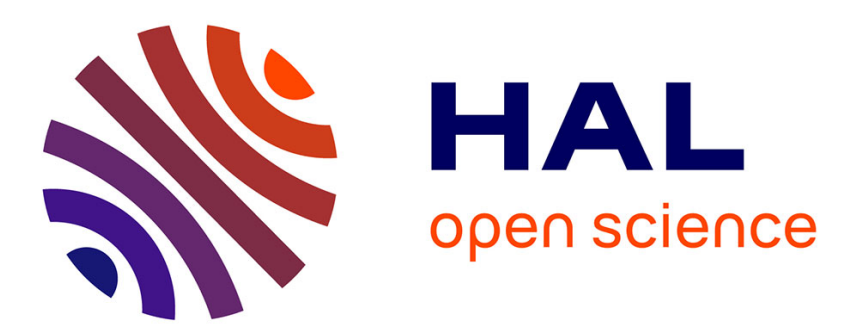

\title{
Identification du rituel dans l'habitat: proposition d'approche à partir de l'exemple d'un tronçon du fossé rhénan (Hallstatt et La Tène)
}

Jonathan Engel

\section{- To cite this version:}

Jonathan Engel. Identification du rituel dans l'habitat: proposition d'approche à partir de l'exemple d'un tronçon du fossé rhénan (Hallstatt et La Tène). Archimède: archéologie et histoire ancienne, 2014, 1, pp.7-20. halshs-01585317

\section{HAL Id: halshs-01585317 https://shs.hal.science/halshs-01585317}

Submitted on 11 Sep 2017

HAL is a multi-disciplinary open access archive for the deposit and dissemination of scientific research documents, whether they are published or not. The documents may come from teaching and research institutions in France or abroad, or from public or private research centers.
L'archive ouverte pluridisciplinaire $\mathbf{H A L}$, est destinée au dépôt et à la diffusion de documents scientifiques de niveau recherche, publiés ou non, émanant des établissements d'enseignement et de recherche français ou étrangers, des laboratoires publics ou privés. 


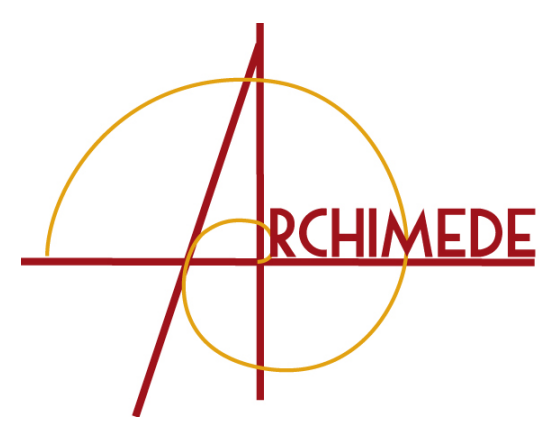

\title{
IDENTIFICATION DU RITUEL DANS L'HABITAT : PROPOSITION D'APPROCHE À PARTIR DE L'EXEMPLE D'UN TRONÇON DU FOSSÉ RHÉNAN (HALLSTATT ET LA TĖNE)
}

\author{
Jonathan ENGEL \\ diplômé du Master « Archéologie du territoire », \\ Université de Strasbourg \\ engel.jonathan@laposte.net
}

RÉSUMÉ

Ces dernières années, l'accumulation des données induite par l'archéologie préventive et la sensibilité accrue des archéologues ont permis la découverte croissante de configurations remarquables de l'âge du Fer souvent perçues comme les vestiges de rituels. La thématique du rituel est peu développée pour le fossé rhénan de l'âge du Fer. Cela nous a incité à constituer un premier inventaire des vestiges potentiellement en lien avec une pratique rituelle inscrite dans I'habitat. Cette recherche s'est fondée sur l'examen de la documentation archéologique, à savoir les rapports de fouilles et les publications faisant état d'un habitat de l'âge du Fer dans un tronçon particulier du fossé rhénan. De tels gisements, altérés par l'érosion, se réduisent à des structures en creux du type fosse, silo, puits, fossé ou encore trous de poteaux, rarement associés à des

Mots-CLÉS

Rituel,

habitat,

âge du Fer,

fossé rhénan,

micro-vase,

jeton,

os humain isolé, dépôt. plans de bâtiment. Cette réalité nous contraint à restreindre le champ d'investigation à l'étude de quelques marqueurs pour proposer un état des lieux sur les indices de pratiques rituelles à l'âge du Fer pour une partie du sillon rhénan.
In recent years the amount of data provided by « rescue archaeology » has increased, as well as archaeologists' awareness. There has followed the discovery of more and more Iron Age features which are often perceived as remains of rituals. The topic of ritual is poorly developed for the Rhine rift valley of the Iron Age. It has therefore encouraged us to establish a provisional inventory of the remains linked potentially to ritual practices in these settlements. This research is based on the examination of the archaeological evidence, namely excavation reports and publications on Iron Age settlements of a specific section of the Rhine valley. Such sites, altered by erosion, reveal no more than hollow-like structures such as pits, silos, wells, ditches or post holes, rarely associated to plans of buildings. As a consequence, our research field was restricted to the study of a limited number of markers in order to take stock of the evidence for Iron Age ritual practices in a segment of the Rhine valley.
KeYwords

Ritual, settlement, Iron Age, Rhine rift valley, miniature vessel, token, isolated human bone, deposit. 
Le rituel est un aspect de la vie des populations de l'âge du Fer de mieux en mieux perçu par les archéologues ces dernières années. L'augmentation des fouilles de sites d'habitat et la sensibilité accrue des chercheurs ont permis d'identifier les traces de pratiques rituelles et des vestiges à portée symbolique au sein de I'habitat, hors des lieux de culte monumentaux [1].

Cette problématique ne fait l'objet de recherches que depuis quelques années, même si auparavant déjà, certains vestiges découverts en contexte d'habitat pouvaient, par leur singularité, être rapprochés du domaine rituel.

Cette contribution [2] présente une première synthèse sur la question des rituels dans I'habitat pour un tronçon du fossé rhénan. La zone d'étude retenue correspond au département du Bas-Rhin diminué des cantons de Sarre-Union et de Drulingen, auquel s'ajoutent les Landkreise (arrondissements allemands) de Rastatt, Ortenau, Emmendingen et le Stadtkreis de Baden-Baden. L'analyse critique des rapports de fouille et des publications concernant quelques centaines de gisements révélant un site d'habitat dans cette zone d'étude a permis de retenir des vestiges provenant de 57 gisements, dont 37 sont occupés au cours du Hallstatt $\left(\mathrm{VIII}^{\mathrm{e}}-\mathrm{VI}^{\mathrm{e}}\right.$ siècle av. J.-C), quatre au cours de la transition entre les deux âges du Fer (autour de 450 av. J.-C.) et 25 au cours de La Tène ( $v^{\mathrm{e}}-\mathrm{I}^{\mathrm{er}}$ siècle av. J.-C).

Les sites d'habitat étudiés n'ayant révélé aucun élément permettant d'identifier des installations particulières liées à l'accomplissement de rituels, ce travail a essentiellement été axé sur l'analyse du mobilier et de son contexte. L'identification de vestiges rituels au sein de I'habitat n'est cependant pas chose aisée. En effet, parmi les vestiges exhumés, le mobilier utilisé au cours d'un rituel et le mobilier domestique ou artisanal ordinaire ne semblent que rarement différenciables. Bien que I'usage de certains vestiges singularisés par leurs propriétés intrinsèques soit perçu comme spécifique pour le rituel, l'objet manipulé dans le cadre d'une telle pratique

[1] Le monde celtique connaît des lieux de culte de formes diverses pour tout l'âge du Fer. Ils sont toutefois plus rares au $\mathrm{I}^{\text {er }}$ âge du Fer et apparaissent sous la forme de sanctuaires fossoyés à partir du III $^{\mathrm{e}} \mathrm{S}$. av. J.-C. (ARCElin \& Brunaux 2003, p. 243-247). Ces derniers sont à ce jour quasiment inconnus dans le fossé rhénan.

[2] Cet article s'appuie sur mon mémoire de Master 2 dirigé par Loup Bernard et soutenu en 2013 à l'université de Strasbourg.

[3] Nous considérons un vestige en position remarquable dès lors qu'il correspond plus souvent à un objet commun détourné de sa fonction première. L'identification d'un rituel au sein de I'habitat est alors envisageable par l'examen de chaque configuration. L'état du mobilier (entier, partiel, manipulé, sélectionné), sa valeur lorsqu'elle est déterminable (économique ou symbolique), sa position (au fond d'une structure, dans le comblement, en bordure de structure, etc.), son association avec d'autres mobiliers, mais aussi la taphonomie (puisqu'elle altère le fait archéologique) constituent les paramètres à prendre en compte pour identifier une configuration remarquable [3]. Néanmoins, la remarquabilité d'un fait n'est pas suffisante pour identifier un vestige de pratique rituelle. Ce niveau d'interprétation devient pertinent lorsque la forme d'une configuration est observée à de multiples reprises. Cela traduit alors l'itération d'un phénomène qui entre justement, mais non pas de manière exclusive, dans la définition du rituel. Un rituel correspond à des pratiques, profanes ou sacrées, effectuées selon des règles précises qui sont reproduites fidèlement dans le temps. Ainsi, pour les vestiges conservés d'un même type de rituel, nous devrions observer des constantes dans la forme des configurations. C'est pourquoi, à la suite d'une étude au cas par cas (Engel 2013) [4], nous avons tenté d'approcher le domaine rituel en regroupant chaque fait retenu dans notre corpus par catégorie de mobiliers ou «marqueur », pour tenter d'identifier des récurrences dans la forme des configurations.

Le dépouillement des différents rapports de fouille nous a permis de prendre connaissance des inhumations en silo, qui ne sont pas prises en compte dans cette étude bien qu'une attention particulière mériterait de leur être portée. Les travaux menées par Valérie Delattre et ses collaborateurs dans le secteur de la confluence SeineYonne semblent démontrer que ces inhumations ne sont pas de simples sépultures de relégation, mais refléteraient plutôt des pratiques cultuelles ou des rites funéraires [5]. Un état de la question pour l'Alsace a d'ailleurs été réalisé récemment [6].

se trouve au fond d'une fosse, en bordure de structure, qu'il est mis en scène ou encore qu'il apparaît au même niveau que d'autres vestiges singuliers.

[4] L'analyse au cas par cas de chaque configuration retenue est disponible sous la forme d'une base de données sur le web-SIA ArkeoGIS - www.arkeogis.org (BERNARD 2014).

[5] Delattre \& Séguier 2005, p. 252.

[6] LANDOLT et al. 2010, p. 220-226 ; FLEISCHER et al. 2013. 


\begin{tabular}{|c|c|c|c|c|c|c|c|c|c|c|c|c|c|}
\hline \multirow[b]{2}{*}{$\mathbf{N}^{\circ}$} & \multirow[b]{2}{*}{ Gisement } & \multicolumn{12}{|c|}{ Catégories de mobilier } \\
\hline & & 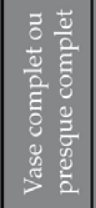 & 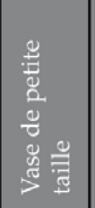 & 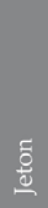 & 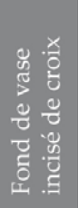 & 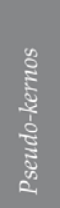 & 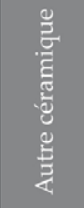 & 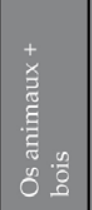 & 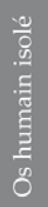 & 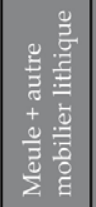 & 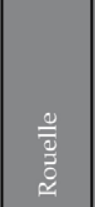 & 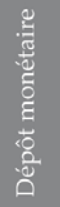 & 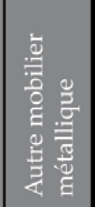 \\
\hline 01 & Achenheim, Ziegelhof - Ancienne loessière Schaeffer & $X$ ? & $\mathrm{X}$ & & & & & & & $X^{\text {Ass_1 }}$ ? & & & \\
\hline 02 & Altorf, Lange Straenge & $X^{\text {Ass_1 }}$ & & & & & & & & $X^{\text {Ass_1 }}$ & & & \\
\hline 03 & Benfeld, Parc d'activités économiques des Nations & & & & & & $X^{\text {Ass_1 }}$ & $\mathrm{X}^{\text {Ass_1 }}$ & & & & & \\
\hline 04 & Bourgheim, 99, rue de Zellwiller & & & $\mathrm{X}$ & & & & & & & & & \\
\hline 05 & Brumath, Lotissement Edouard Manet - Betzetweg & & $\mathrm{X}$ & & & & & & & & & & \\
\hline 06 & $\begin{array}{l}\text { Duntzenheim, Ebenheit - LGV Est, Stock nord, secteur 2, } \\
\text { tranche } 1\end{array}$ & $\mathrm{X}^{\text {Ass_1 }}$ ? & & & & & & & & $\mathrm{X}^{\text {Ass_1 }}$ ? & & & \\
\hline 07 & Eckbolsheim, Parc d'Activités & $X^{\text {Ass_1 }}$ ? & $\begin{array}{c}X \\
X^{\text {Ass_1 }} \text { ? }\end{array}$ & & & & & & & & & & \\
\hline 08 & Eckbolsheim, Lotissement - Rue des frères Lumières & & & & & & & & & & $\mathrm{X}^{\text {Ass_1 } 1 ?}$ & & $X^{\text {Ass_1 }}$ \\
\hline 09 & Emmendingen, Brettenbach & & & & & & & & & & & & $x$ \\
\hline 10 & Endingen, Erste und Zweite Strecke & & & & $X$ & & & & & & & & \\
\hline 11 & Endingen, Wilhelmskapelle & & & & & & & $\mathrm{X}$ & & & & & \\
\hline 12 & Entzheim, Im Sellit & & $X$ & & & & & & & & & & \\
\hline 13 & Entzheim, In der Klamm & $X^{\text {Ass_1 }}$ & $\begin{array}{c}X \\
X^{\text {Ass_2 }} \\
X^{\text {Ass_4 ? }}\end{array}$ & & & $\mathrm{X}$ & & $X^{\text {Ass_3 }}$ & & $\begin{array}{l}X^{\text {Ass_1 }} \\
X^{\text {Ass_3 }}\end{array}$ & & & \\
\hline 14 & Eschau, Ban communal & & & & & & & & & & $\mathrm{x}$ & & \\
\hline 15 & Fegersheim, Ohnheim, Lotissement Gentil Home 2000 & $X^{\text {Ass_1 }}$ & & & & & & & & & & & \\
\hline 16 & Forchheim, Heckle & & $X$ & $\mathrm{X}$ & & & & $\mathrm{X}$ & & & & & \\
\hline 17 & Geispolsheim, Schwobenfeld & & $\mathrm{X}$ & & & & & $X^{\text {Ass_1 }}$ & & & & & $x$ \\
\hline 18 & Geispolsheim, Bruechel & $\mathrm{X}^{\text {Ass_1 }}$ & $x$ & $X$ & & & & & & & & & \\
\hline 19 & Gougenheim, Tiergarten, Steinbrunnen & $\mathrm{X}$ & & & & & & & & & & & \\
\hline 20 & Goxwiller, ZAC PAEI de Goxwiller/Valff & & $\mathrm{X}$ & & & & & & & & & & \\
\hline 21 & Hatten, Rothsmatt & & $\mathrm{x}$ & & & & & & & & & & \\
\hline 22 & Herbolzheim, Untere Immele & & $\mathrm{X}$ & & & & & & & & & & \\
\hline 23 & Hindisheim, Borot & & & $\mathrm{X}$ & $\mathrm{X}$ & & & & & & & & \\
\hline 24 & Holtzheim, Am Schluesselberg & & & & & & & $\mathrm{X}$ & & & & & \\
\hline 25 & Jechtingen, Hochberg, Gaisshorn & & & $\mathrm{X}$ & & & & & & & & & \\
\hline 26 & Jechtingen, Lachenmüngle & & $\mathrm{X}$ & & & & & & & & & & \\
\hline 27 & Kienheim & & $\mathrm{X}$ & & & & & & & & & & \\
\hline 28 & La Wantzenau, Lotissement Kirchacker & & & & & & & & & & & & $X^{\text {Ass_1 } 1 ? ~}$ \\
\hline 29 & Lingolsheim, Sablières Modernes & & & $X$ & $\mathrm{X}$ & & & & & & & & \\
\hline 30 & Marlenheim, Contournement routier & & & & & & & & & $x$ & & & \\
\hline 31 & Marlenheim, Hofstatt & & $\mathrm{X}$ & $\mathrm{X}$ & & & & & & & & & \\
\hline 32 & Marlenheim, Lotissement «le Domaine de la Couronne d'Or» & & $\mathrm{X}$ & & & & & $\mathrm{X}$ & & & & & \\
\hline 33 & Marlenheim, Maison Apprederis, Impasse du Stift & & $\mathrm{X}$ & $\mathrm{X}$ & & & & & $X$ & & & $x$ & \\
\hline 34 & Meistratzheim, Station d'épuration intercommunale, Pfett & \begin{tabular}{c|c}
$X$ \\
$X^{\text {Ass_ } 1}$
\end{tabular} & & & & & & $\begin{array}{c}X \\
X^{\text {Ass_1 }} \\
X^{\text {Ass_2 }} \\
X^{\text {Ass_3? }} \\
\end{array}$ & & & & & \\
\hline 35 & $\begin{array}{l}\text { Mittelschaeffolsheim, Gute Abwand, } \\
\text { LGV Est, Tronçon H - Site } 10.4\end{array}$ & & & & & $\mathrm{X}$ & & & & & & & \\
\hline 36 & Mundolsheim, RMS - rue Ampère & & $\mathrm{X}$ & & & & & & & & & & \\
\hline 37 & Mundolsheim, Strengfeld & & $\mathrm{X}$ & & $\mathrm{X}$ & & & & & & & & \\
\hline 38 & Oberschopfheim, Rütte & & $\mathrm{X}^{\text {Ass_1 }}$ & & & & & & & $X^{\text {Ass_1 }}$ & & & \\
\hline 39 & Ottrott, Mont Sainte-Odile & & & $X$ & & & & & & & & & \\
\hline 40 & Pfulgriesheim, Lotissement communal, Langgarten et Buetzel & & $\mathrm{X}$ & & & & $\mathrm{x}$ & $\mathrm{x}$ & & & & & \\
\hline
\end{tabular}




\begin{tabular}{|c|c|c|c|c|c|c|c|c|c|c|c|c|c|}
\hline \multirow[b]{2}{*}{$\mathbf{N}^{\circ}$} & \multirow[b]{2}{*}{ Gisement } & \multicolumn{12}{|c|}{ Catégories de mobilier } \\
\hline & & 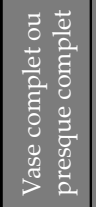 & 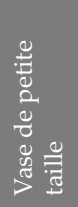 & $\stackrel{\Xi}{\varrho}$ & 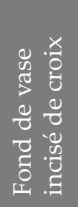 & 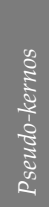 & 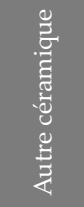 & 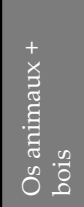 & 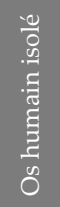 & 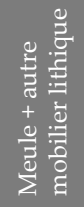 & $\begin{array}{l}\stackrel{0}{\bar{\Xi}} \\
\tilde{0} \\
\stackrel{0}{ \pm}\end{array}$ & 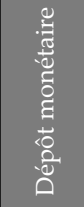 & 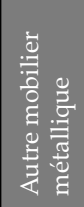 \\
\hline 41 & Riegel, Romansbuck, Rumlisbuck & & $x$ & & & & & & & & & & \\
\hline 42 & Riegel, Lindenbrunnenbuck & & $x$ & & & & & & & & & & \\
\hline 43 & Riegel, Fronhofbuck & $\mathrm{X}^{\text {Ass_1 }}$ & & & & & & & & & & $X^{\text {Ass_1 }}$ & \\
\hline 44 & Rosheim, Leimen & & $X$ & $x$ & & & & & & $\mathrm{X}$ & & & \\
\hline 45 & Rosheim, Parc du Rosenmeer, Strasbourg Câbles & & & & & & & & & $x$ & & & \\
\hline 46 & Rosheim, Sandgrube & & & & & & & & & & & & $x$ \\
\hline 47 & Rosheim, Parc d'Activité du Rosenmeer, Laser Alsace & $\mathrm{X}^{\text {Ass_1 }}$ & & & & & & $X^{\text {Ass_-1 }}$ & & & & & \\
\hline 48 & Rosheim, Sablière Helmbacher & & & & & & & & $X$ & & & & \\
\hline 49 & Rosheim, Mittelweg & & & & & & & $X$ & $x$ & & & & \\
\hline 50 & Sasbach, Limberg & $\mathrm{X}$ & $X$ & & & & & & $x$ & & & & \\
\hline 51 & Sasbach, Schafläger, Hirschländer & $x$ & & & & & & & & & & & \\
\hline 52 & Saverne, Fossé des Pandours, Attaque des Pandours & & & & & & & & & & $X$ & & \\
\hline 53 & Saverne, Fossé des Pandours, Barbarakopf Zone 3 & $X^{\text {Ass_1 } 1 ? ~}$ & & $x$ & & & $X^{\text {Ass_1 } 1}$ & & & $X^{\text {Ass_1 } 11}$ & & & $\mathrm{X}^{\mathrm{Ass} \_1}$ ? \\
\hline 54 & Saverne, Fossé des Pandours, Barbarakopf Zone 9 & $\begin{array}{c}X \\
X^{\text {Ass_2 }}\end{array}$ & & $x$ & & & & & & $\mathrm{X}^{\mathrm{Ass} \_} \mathrm{L}_{2}$ & & & $X^{\text {Ass_2 } 2}$ \\
\hline 55 & Saverne, Fossé des Pandours, Murus Gallicus & & & & & & & & & & & $X$ & \\
\hline 56 & Schaeffersheim, Lotissement communal «La Chênaie» & & & & & & & $X$ & $X$ & $x$ & & & \\
\hline 57 & Valff, Blasiusfeld & & & $X ?$ & $x$ & & & & & & & & \\
\hline 58 & Westhouse, Ziegelhof & & $X$ & & & & & & & & & & \\
\hline 59 & Wiwersheim, ZA du Kochersberg & $X^{\text {Ass_1 }}$ & & & & & & & & $X^{\text {Ass_1 } 1}$ & & & \\
\hline 60 & Wolfisheim, Westermatt & $X^{\text {Ass_1 }}$ & $\mathrm{X}$ & $X$ & & & & & & & & & \\
\hline
\end{tabular}

Légende : X $X^{\text {Ass_0 }}$ Mobilier en association

Les lignes suivantes présenteront pour chaque marqueur un état de la question en mettant l'accent sur les constantes chronologiques et morphologiques des configurations, pour ensuite proposer une démarche visant à approcher le domaine rituel.

\section{LES MARQUEURS [7]}

Un type de mobilier a été retenu comme «marqueur » dès lors qu'il intégrait une configuration remarquable, qu'il était découvert, à l'échelle du monde celtique, en contexte cultuel [8] ou qu'il était rattaché au domaine symbolique et rituel pour ses caractéristiques intrinsèques ou par un chercheur. Les gisements mentionnés ci-après sont référencés en figure 1.

Les « marqueurs » retenus ici ne sont évidemment pas exhaustifs. La sensibilité particulière d'un chercheur ou les avancées méthodologiques et techniques seront en mesure de développer de nouvelles approches susceptibles d'enrichir le débat sur les pratiques rituelles de l'âge du Fer.

\section{Les vases complets}

D'après les critères énoncés ci-dessus, plusieurs configurations comprenant des céramiques ont été relevées. La catégorie des vases complets est également reconnue par F. Gransar et ses collaborateurs [9] pour l'identification de manifestations rituelles.

Au total, 18 configurations ont été recensées dans la zone d'étude [10] (Fig. 2). À première vue, leur composition ne semble présenter que peu de constantes. Toutefois, deux types de configurations se répètent à plusieurs reprises : trois occurrences pour la catégorie « vase entier sans association en position non-remarquable » (Sasbach Limberg et Sasbach Schafläger), deux pour la catégorie «vase quasi entier sans association en position remarquable »

[7] Les jetons en céramique et les fonds de vase incisés sont des marqueurs pris en compte pour cette étude, mais non décrits ciaprès.

[8] Nous entendons par contexte cultuel tout lieu de culte de I'âge du Fer en Europe tempérée ou tout fait issu de la même aire chronoculturelle, interprété comme symbolique ou rituel.

[9] Gransar et al.. 2007, p. 559, fig. 12.

[10] ENGEL 2013, vol. I, p. 66-72. 
(Gougenheim Tiergarten, Ottersthal Fossé des Pandours). En observant la composition des associations un peu plus en détail, nous relevons en plus les constantes suivantes : neuf configurations du type «assemblage de plusieurs vases » et quatre configurations du type «vase associé à au moins une ou plusieurs meule(s) ».

L'analyse des données (Fig. 2) fait ressortir une disparité des formes de chaque configuration. De ce fait, il est peu probable que les gestes à leur origine soient tous semblables. À côté des configurations peu pertinentes pour alimenter une réflexion sur la question des rituels dans I'habitat, comme l'ensemble « vase entier sans association en position non-remarquable », il y en a également d'autres, plus intéressantes. Par exemple, l'ensemble «vase entier associé à d'autres vases, des meules et des outils métalliques en position remarquable » (Ottersthal, Fossé des Pandours [11]) traduit, quant à lui, un véritable dépôt intentionnel, vraisemblablement constitué à la suite d'un rituel.

\section{Les micro-vases et les petits vases}

Nous distinguons deux catégories de vases de petite taille en fonction de leurs dimensions [12] : les « microvases » et les «petits vases» (Fig. 3). Les fonctions proposées pour ces petits objets sont multiples et variables selon le contexte [13]. À titre indicatif, voici les plus fréquentes: usage rituel dans la sphère domestique, traitement du métal [14], usage dans les sanctuaires. Des traces de substances hallucinogènes, de boissons fermentées, de restes d'encens et de substances colorantes, révélées par des analyses chimiques effectuées sur des individus semblables à Pontos [15] (Ampurdan, Espagne) seraient pour les chercheurs des indices en faveur d'un usage rituel.

Notre corpus [16] compte 90 individus découverts sur 26 gisements pour l'ensemble de l'âge du Fer. Le nombre de vases de petite taille, élevé au premier âge du Fer, diminue considérablement au second âge du Fer (Fig. 4). 66 individus sont datés du Hallstatt, 16 de la période de transition entre les deux âges du Fer, cinq de La Tène ancienne (480 à 280 av. J.-C.) et seuls deux proviennent d'un gisement de La Tène finale (150 à 30 av. J.-C).

Une interprétation de ces vases, évoquée dans le rapport de fouille d'Entzheim In der Klamm [17], nous a rendu attentif à une association bien particulière. En effet, la proximité des vases de petite taille avec du mobilier de tissage ou de filage pourrait indiquer leur utilisation en tant que mouilloir dans le cadre d'activités de filage. Parmi les structures ayant contenu des vases de petite taille, $43 \%$ ont livré par ailleurs des fusaïoles, des pesons ou des bobines.

Au final, la grande majorité des habitats concernés par ces individus sont occupés au cours du premier âge du Fer et plus rarement au début du second. Ces petits vases sont essentiellement mêlés à des détritus de nature domestique et ne témoignent d'aucune attention particulière au moment de leur abandon - une attention que l'on pourrait attendre pour des objets sacrés. Souvent fragmentés ou entiers, ils apparaissent quelquefois en nombre appréciable à l'échelle d'un site ou encore à l'échelle d'une même structure, sans qu'aucune forme préférentielle ne soit, à première vue, identifiée pour les individus issus des contextes les plus remarquables. Toutefois, le fort taux d'association de ces vases à du mobilier de tissage/filage pourrait indiquer une utilisation comme mouilloir pour le filage.

[11] ODRY \& FÉLIU 2009, p. 276-277.

[12] Cette classification s'inspire de la définition des vases de petite taille proposée par H. van den Boom (VAN DEN BOom 1989, p. 2934).

[13] AdAM et al. 2011, p. 62.

[14] ROTTLÄNDER 1985, p. 19.

[15] ROPIOT \& MAZIÈRE 2007, p. 743.

[16] ENGEL 2013, vol. I, p. 73-84.

[17] Landolt et al.. 2013 p. 94, 267 [inédit].

Figure 2:

Graphique : nombre des configurations incluant des vases en fonction de leur état et de leur position. Engel 2013, vol. I, p. 71

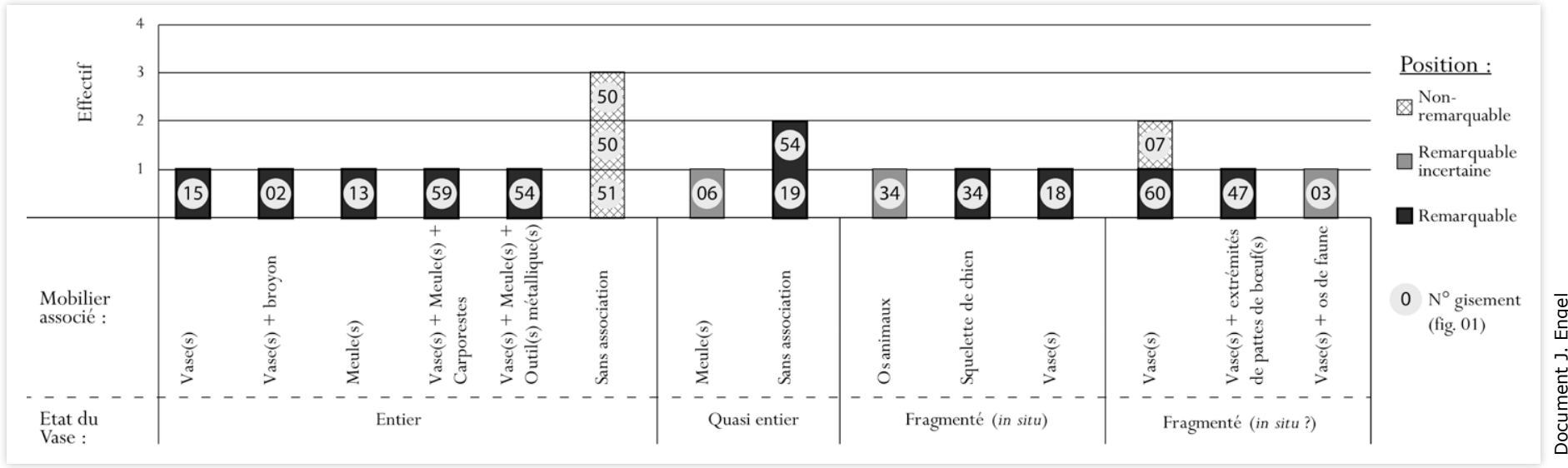




\begin{tabular}{|l|l|l|}
\cline { 2 - 3 } \multicolumn{1}{c|}{} & $\begin{array}{l}\text { Micro-vase } \\
\text { (Kleinstgefäss) }\end{array}$ & $\begin{array}{l}\text { Petit vase } \\
\text { (Kleingefäss) }\end{array}$ \\
\hline Forme haute & $\begin{array}{l}\text { Hauteur inférieure } \\
\text { à } 5 \mathrm{~cm}\end{array}$ & $\begin{array}{l}\text { Hauteur comprise } \\
\text { entre } 5 \text { et } 10 \mathrm{~cm}\end{array}$ \\
\hline Forme basse & $\begin{array}{l}\text { Diamètre inférieur } \\
\text { à } 5 \mathrm{~cm}\end{array}$ & $\begin{array}{l}\text { Diamètre compris } \\
\text { entre } 5 \text { et } 10 \mathrm{~cm}\end{array}$ \\
\hline
\end{tabular}

Figure 3 :

Définition des micro-vases et des petits vases. Engel 2013, vol. I, p. 45.

\section{La faune}

Les restes osseux constituent un « marqueur » préférentiel pour l'identification d'un rituel [18]. Leur état de conservation, leur disposition, leurs éventuelles traces de découpe, l'état complet ou partiel (part sélectionnée) du squelette, etc., permettent souvent de reconstituer les gestes à l'origine d'un dépôt de faune. Pour cette étude, ont essentiellement été retenus les squelettes entiers, les parties de squelettes en connexion anatomique, les crânes ou encore les bois de cervidés.

17 configurations mises au jour sur 12 gisements ont été enregistrées [19]. De manière générale, chaque période est représentée par un nombre relativement équivalent de ce type de configurations. Le graphique en figure $\mathbf{5}$ illustre la diversité des configurations retenues en tenant compte de l'espèce animale, des éléments anatomiques concernés et des vestiges associés. En considérant uniquement l'espèce, nous avons enregistré, pour le bœuf, trois configurations, quatre pour le cheval, six pour le chien, une pour le porc et deux pour le cerf. Sans effectuer un recensement complet à partir des éléments anatomiques, nous dénombrons trois crânes entiers (deux de bœuf et un de cheval), deux configurations ayant contenu un bois de cerf complet, une autre caractérisée par plusieurs ensembles en connexion anatomique et également sept ensembles formés d'au moins un squelette complet. Cette diversité est également perceptible au niveau des associations de vestiges. Effectivement, huit configurations ne présentent aucune association, deux correspondent à des associations de même nature, deux à des associations d'os animaux provenant d'espèces différentes, trois à des associations incluant de la céramique et une à une association avec une meule.

La catégorie des os animaux semble effectivement caractérisée par la diversité des configurations, en matière de composition. Les squelettes entiers peuvent autant correspondre à un dépôt d'offrande succédant à un sacrifice qu'à une mise en terre de la dépouille d'un animal arrivé au terme de sa vie, sans que sa mort n'ait nécessairement été provoquée dans le cadre d'un rituel. Les ensembles d'os en connexion anatomique sont plus intéressants sous l'angle rituel. Ils témoignent de la présence de chairs autour des os au moment de leur enfouissement et peuvent avoir fait I'objet d'une sélection. Il est entendu que les pièces d'un faible intérêt culinaire peuvent correspondre à des déchets de boucherie. Toutefois, lorsqu'il s'agit de parties « nobles » ou lorsque d'autres indices s'ajoutent à ces ensembles, l'interprétation en faveur d'un rituel est plus vraisemblable. Quant aux crânes, lorsqu'ils sont découverts sans autres déchets de boucherie, ils sont le résultat de prélèvements des têtes animales qui ont alors pu bénéficier d'un traitement particulier, différent du reste des corps, en liaison peut-être avec une valeur symbolique et/ou pour être exposés.

[18] BRunaux 2000, p. 134.

[19] ENGEL 2013, vol. I, p. 92-100.

Figure 4 :

Graphique : nombre de «vases de petite taille» par catégorie et par période chronoculturelle. Engel 2013, vol. I, p. 82.

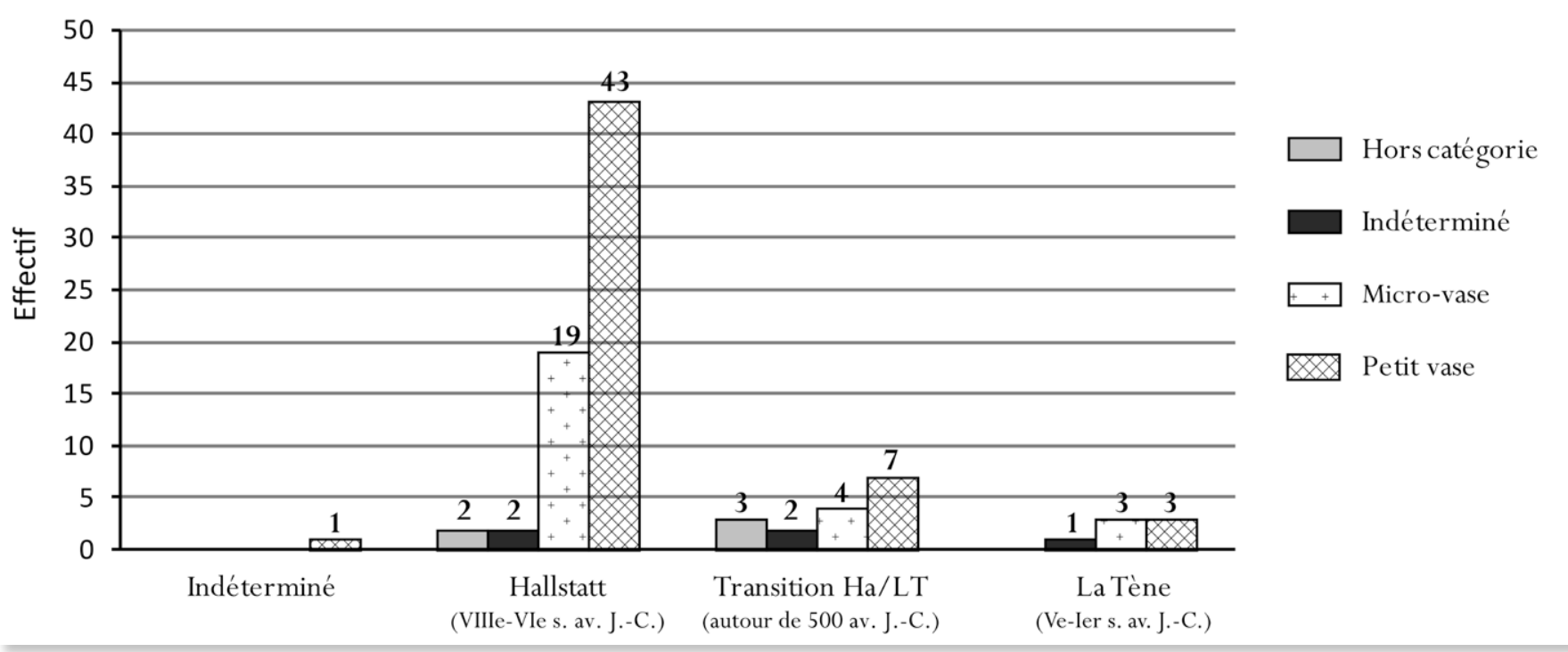



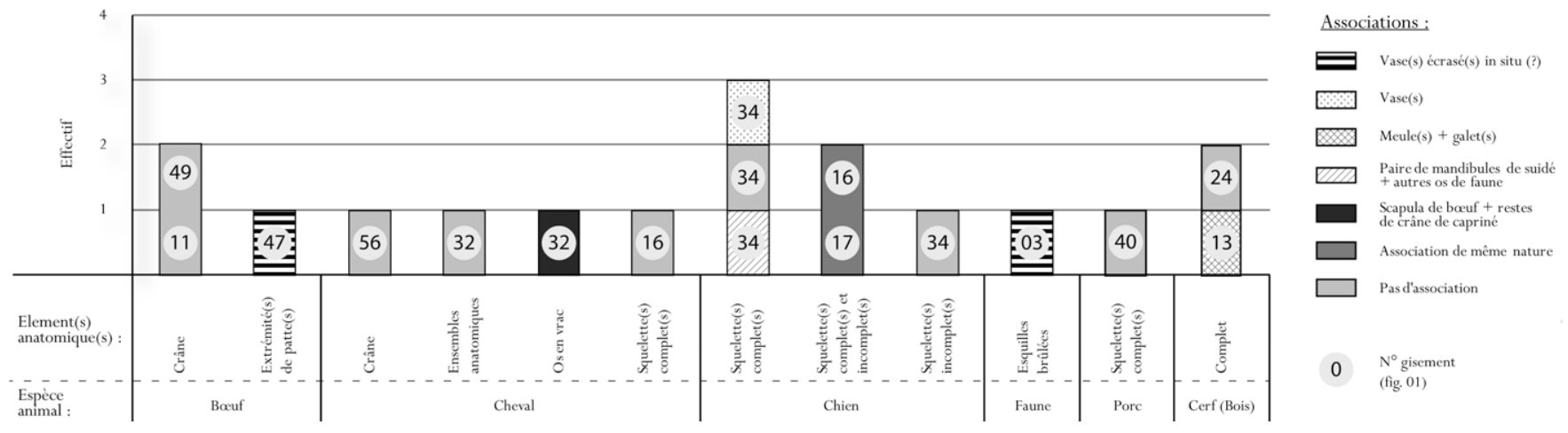

Ainsi, le graphique ne laisse apparaître que peu de constantes. Ce constat est certainement dû à l'effectif réduit de notre corpus. Il convient tout de même de mentionner le nombre relativement important de squelettes de chiens. Neuf sujets ont été dénombrés. Ils ont pour la plupart été découverts de manière isolée dans des fosses. Est-ce que ces chiens ont ici bénéficié d'une attention particulière après leur mort ? Sont-ils l'objet d'un dépôt d'offrande ? En l'absence d'indices, il est bien souvent difficile de le déterminer. Deux configurations regroupent plusieurs individus, comme la fosse n 5011 de Geispolsheim Schwobenfeld qui en contenait trois [20]. Dans ces cas, la mort des chiens semble relativement synchrone, et par extrapolation, il est probable qu'elle ait été provoquée intentionnellement.

\section{Les os humains isolés}

Si la présence d'os humains dans les sépultures est normale, leur découverte de manière isolée dans les habitats est plus surprenante. Pourtant, ces cas sont assez fréquents au cours de La Tène [21]. Valérie Delattre et Jean-Marc Séguier énumèrent les différents lieux de mise au jour de ces os isolés dans le secteur de la confluence Seine-Yonne : «les sites à vocation cultuelle et collective de la fin de l'âge du Fer », « les nécropoles » et « les établissements ruraux des deux derniers siècles avant J.-C. »[22]. La signification de ces ossements en contexte domestique reste toutefois difficile à percevoir. Cependant, comme l'indiquent les deux chercheurs, leur éventuelle association avec du mobilier atypique permet plus aisément de le rattacher au domaine cultuel [23].

[20] LANDOLt et al.. 2010, p. 223.

[21] L'oppidum de Manching a par exemple livré près de 5000 os humains isolés (LANGE 1983, p. 3).

[22] Delattre \& Séguier 2007, p. 615.

[23] Ibid. p. 616.

[24] ENGEL 2013, vol. I, p. 101-103.
La présence d'os longs dans I'habitat peut également s'expliquer par l'action des charognards sur le squelette de défunts, comme celle des chiens. Dans ce cas, des traces de morsures devraient pouvoir le déterminer. Cette explication pragmatique ne peut pourtant pas être étendue à l'ensemble de ces faits. Il est peu vraisemblable que l'action des chiens ait été plus intense à la période laténienne, celle pendant laquelle les os humains se rencontrent fréquemment dans I'habitat.

Dans notre corpus, sept gisements sont concernés par la présence d'os humains isolés [24]. Concernant la répartition chronologique, deux gisements sont datés de La Tène ancienne et cinq de La Tène finale. Lorsque I'on considère le type d'os découvert dans I'habitat, on constate que seuls les fragments de crânes, au nombre de cinq, et les fémurs, au nombre de trois, sont représentés. Des cas similaires sont observés par V. Delattre et J.-M. Séguier ; là aussi, seuls des os longs et des crânes, fragmentés ou non, sont découverts en contexte d'habitat [25].

Finalement, le contexte de découverte des os isolés ne permet pas de préciser leur emploi au sein de I'habitat. Toutefois, tout comme dans d'autres régions, il semblerait que les os longs et les fragments de crânes soient relativement plus importants dans les habitats du second âge du Fer, ce qui semble traduire une pratique particulière.

\section{Les meules en dépôt}

Seules les meules (plus un individu hors de cette catégorie [26]) pouvant par leur position, leur état [27] ou le

[25] Exemple de l'établissement rural d'Herblay (Val d'Oise) (DeLATTRE \& SÉGUIER 2007, p. 615).

[26] Il s'agit d'une grande pierre en grès hémisphérique associée à deux petits vases (Oberschopfheim Rütte, Ortenau Kreis) qui n'est pas une meule (ENGEL 2013, vol. II, p. 190-192).

[27] Ces meules sont entières ou quasi entières, avec ou sans traces d'utilisation. 


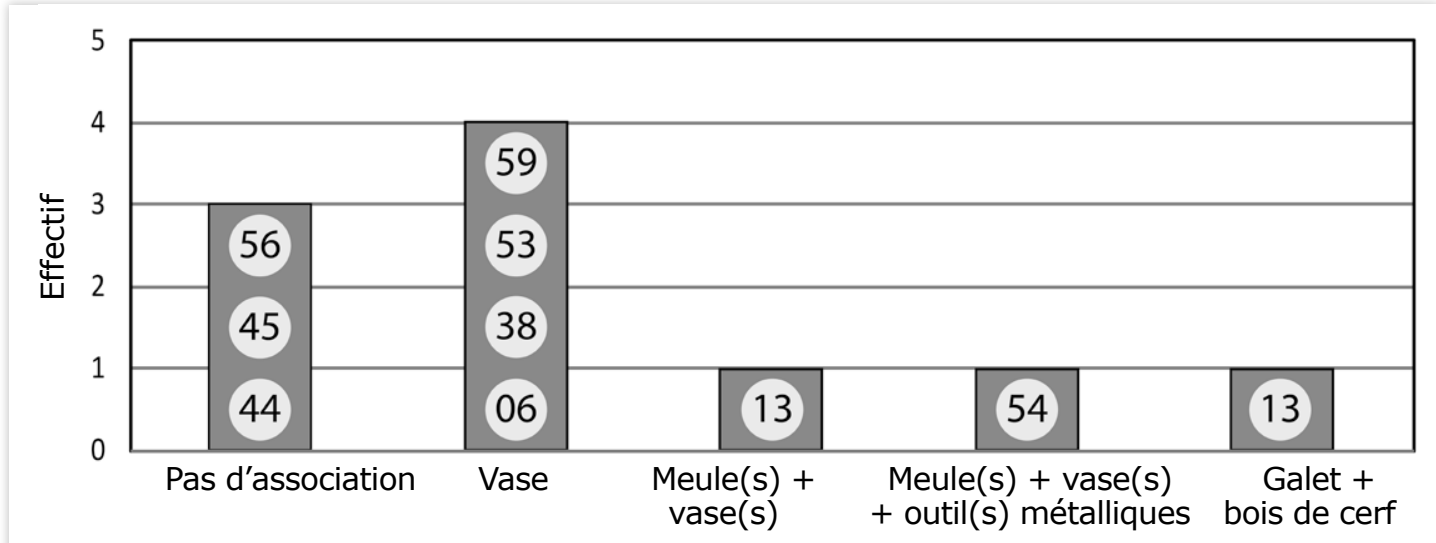

Figure 6

Graphique : effectif des vestiges lithiques par type d'association. Engel 2013, vol. I, p. 108.

$0 \quad \mathrm{~N}^{\circ}$ de gisement (fig. 1)

Document J. Engel

mobilier associé évoquer un dépôt intentionnel ont été retenues.

Dix configurations provenant de huit gisements ont été enregistrées en raison de la présence d'un vestige lithique remarquable. Trois gisements sont datés du premier âge du Fer et cinq du second. Des constantes semblent apparaître au niveau des associations (Fig. 6). En effet, six configurations sur les 11 retenues sont caractérisées par l'association d'un vestige lithique et d'un ou plusieurs vases. Parmi ces six configurations, les deux ensembles découverts dans les puits $n^{\circ} 1$ et $n^{\circ} 3$ de l'oppidum du Fossé des Pandours se composaient de plus d'une meule. Les quatre configurations sans association ont été enregistrées parce qu'elles montraient une éventuelle position remarquable ou un bon état de conservation. La pauvreté des indices relevés pour ces dernières permet difficilement de reconnaître des gestes rituels. L'association meule(s)-vase(s) ne semble pas être spécifique à une période précise, puisque ce type de configuration est relevé dès le Hallstatt $D$ à Entzheim In der Klamm [28], mais également à La Tène $D$ comme à Wiwersheim $Z A$ du Kochersberg [29].

Au final, à l'exception des quatre meules sans association, pour lesquelles il est difficile d'identifier une configuration singulière pouvant résulter d'une pratique rituelle, les autres faits semblent correspondre à des dépôts volontaires, pour lesquels une signification symbolique est envisageable.

\section{Les rouelles}

Les rouelles sont de petites roues de quelques centimètres de diamètre. Elles sont formées d'un nombre variable de rayons reliant la jante au moyeu central et peuvent être réalisées en plomb, en alliage cuivreux, plus rarement en argent et en or. La fonction ou l'utilisation de ces objets a suscité de multiples interprétations. Claude Salicis présente un état de la question dans ses travaux consacrés aux rouelles [30]. Les interprétations les plus fréquemment admises sont : la roue votive, la pré-monnaie ou l'amulette. D'autres interprétations sont évoquées, mais semblent peu probables selon I'auteur. Il s'agit de la rouelle en tant que fusaïole, agent de suspension, boucle de ceinture ou encore en tant que bouton. La valeur symbolique de ces petites roues semble être confirmée par leur présence, quelquefois en très grand nombre, dans les sanctuaires. Plusieurs dizaines de milliers ont été découvertes dans le sanctuaire des Grèves à LaVilleneuve-au-Châtelot [31]. Les rouelles sont également fréquentes sur les oppida de la fin de l'âge du Fer. Christian Peyre attribue à celles de Villeneuve-SaintGermain la fonction de jeton de vote [32], en raison de leur concentration à proximité de structures de type « galerie », peut-être à vocation publique.

Notre corpus compte un total de quatre rouelles découvertes sur trois gisements (Eschau ban communal [33], Eckbolsheim Lotissement [34], Saverne Fossé des Pandours [35]). À l'exception de l'exemplaire d'Eckbolsheim retrouvé dans le comblement inférieur d'un puits, les autres rouelles ne semblent pas présenter de lien avec une structure. Aucun élément permettant de clarifier la fonction de ces objets n'a été relevé.

[28] FLEISCHER \& LANDOLT 2012, p. 198-199.

[29] JODRY \& FÉLIU 2009.

[30] SALICIS 1996, p. 21-38.

[31] Depeyrot \& Piette 2008, p. 58-91.

[32] PeYRe 2000, p. 165, 173.

[33] NoRMAND 1973, p. 82.

[34] KUHNLE et al. 1998, p. 47-48.

[35] FichtL \& AdAM 1999, p. 151, pl. 12 [inédit]. 


\section{La monnaie en dépôt}

Les monnaies ont été recensées lorsqu'elles ont été découvertes dans une position suggérant un dépôt volontaire. Si la présence de monnaies dans les sanctuaires est habituellement le résultat d'une action symbolique, il existe également des dépôts hors de ces aires sacrées, pour lesquels l'hypothèse de l'offrande est formulée [36].

Trois configurations ont été enregistrées sur trois sites occupés à La Tène finale: Riegel Fronhofbuck [37], Marlenheim Maison Apprederis [38] et Saverne Fossé des Pandours [39]. Le premier ensemble correspond à un trésor formé de 27 monnaies imitant les statères de Philippe II de Macédoine. Ces pièces, composées d'un alliage d'or, d'argent et de cuivre, furent retrouvées avec un conteneur en céramique dans un niveau d'occupation daté de La Tène finale. Parmi ces monnaies, 16 ont été éparpillées sur une longueur de $3 \mathrm{~m}$, vraisemblablement suite à l'implantation d'une voie romaine. Quant à la signification de ce dépôt, les auteurs proposent de voir dans cet ensemble une cachette ou un dépôt cultuel. La deuxième configuration a été mise au jour au sein d'un habitat rural. Il s'agit de 12 potins du type dit «au sanglier » découverts dans la fosse $n^{\circ} 150$ localisée dans le secteur des puits. La concentration de ces potins pourrait indiquer qu'ils étaient regroupés dans un contenant en matière périssable. Ici aussi, même s'il est difficile de trancher, I'hypothèse d'une cachette ou d'un dépôt cultuel peut être proposée. La dernière configuration concerne une monnaie découverte à la base du rempart, au nord-ouest de l'oppidum du Fossé des Pandours. Le statère boïen en or dit «Muschelstater » se trouvait entre les gros blocs de pierre qui formaient I'assise du rempart. Selon Stephan Fichtl, il pourrait s'agir d'un dépôt de fondation [40].

\section{LE «POTENTIEL SYMBOLIQUE »}

L'estimation du potentiel de chaque configuration à être lié à un rituel exécuté dans I'habitat de l'âge du Fer prend tout son sens lorsque I'on considère I'hétérogénéité des configurations retenues et les difficultés inhérentes à la lecture et à l'interprétation d'un fait archéologique.

[36] GRUEL 2007, p. 710. Les concentrations de monnaies peuvent également résulter d'une perte accidentelle ou être formées à des fins économiques, comme c'est le cas des cachettes ou des trésors accumulés progressivement ou rapidement.

[37] DeHn \& DrauschKe 2001, p. 82-85.

[38] ChÂTELET et al. 2006, p. 59, 67-69 [inédit].

\section{Introduction à la démarche}

Les vases de petite taille, les jetons en céramique, les fonds de vases incisés font partie du mobilier pour lequel il est bien difficile de statuer sur leur emploi passé. Les configurations remarquables, bien que singulières, présentent une grande diversité dans leur forme (association des objets entre eux et types de contexte). Un vase retrouvé quasi entier au fond d'une structure en creux peut autant avoir été déposé volontairement à des fins symboliques que rejeté car jugé désuet. Plusieurs ensembles d'os animaux en connexion anatomique, sélectionnés et mis en scène, reflètent plus volontiers une pratique rituelle. Les incertitudes induites par les difficultés de lecture et d'interprétation des faits archéologiques, mais aussi les disparités évoquées ci-dessus, nous ont poussé à introduire la notion de «potentialité ». Puisqu'il est souvent impossible de qualifier incontestablement une configuration de rituelle, il est préférable de proposer pour celle-ci un lien potentiel avec une pratique symbolique. De plus, les vestiges retenus pour notre étude ne peuvent être placés sur un même niveau. C'est pourquoi, il nous semble adapté d'estimer le potentiel symbolique de chaque configuration ayant pu résulter d'une activité rituelle par une gradation de valeurs : «faible», «moyen » et « fort ».

\section{Méthode d'attribution d'une « valeur symbolique »}

L'examen d'un phénomène aussi complexe qu'un rituel doit s'effectuer à l'échelle d'un fait. Le mobilier seul, à quelques exceptions près, est souvent trop peu parlant pour ces questions. Le diagramme en figure $\mathbf{7}$ illustre la procédure suivie pour l'attribution des « valeurs symboliques ». Nous distinguons trois voies pour cette estimation selon la catégorie de mobilier concernée :

- le mobilier potentiellement détourné pour un usage rituel: les vases en céramique, les os animaux, les meules, les monnaies, etc. ;

- le mobilier «symbolique » ou à probable usage rituel: les rouelles, les pseudo-kernoi [41], les os humains isolés, etc. ;

- le mobilier multi-usage et difficile à interpréter : les vases de petite taille, les jetons, les fonds de vases incisés, etc.
[39] FICHTL 1996, p. 193-195.

[40] V. note précédente.

[41] Les pseudo-kernoi sont des céramiques qui possèdent, sur leur partie supérieure, plusieurs petits récipients ou cupules appelées kotiliskoi. Ils se distinguent des kernoi par l'absence de perforation faisant communiquer les kotiliskoi et le vase-mère (DELNEF 2006), (LANDOLT \& FLEISCHER à paraître). 
Pour le mobilier détourné, l'estimation de la valeur symbolique d'une configuration requiert la prise en compte de la position du vestige (remarquable ou non), de son état (complet ou fragmenté) et de son association éventuelle avec d'autres vestiges. Nous considérons un vestige en position remarquable dès lors qu'il se trouve au fond d'une fosse, en bordure de structure, qu'il est mis en scène ou encore qu'il apparaît au même niveau que d'autres vestiges singuliers. Ainsi, en fonction de la combinaison de ces paramètres, une structure peut obtenir la valeur «faible», «moyen » ou «fort». L'intitulé de chacune de ces trois valeurs indique le degré de potentialité selon lequel un fait ou un gisement peut être concerné par une pratique rituelle. Il est important de noter que la valeur «fort » n'est pas attribuée à des vestiges de rituels identifiés de manière indéniable. En revanche, pour cette valeur, nous estimons que l'interprétation en faveur d'une pratique rituelle est fortement probable.

La simple présence d'un vestige correspondant à la seconde classe énoncée ci-dessus, même dans un état détritique, autorise la valeur « moyen ». Si d'autres éléments viennent renforcer le faisceau d'indices comme sa position ou des associations, alors la configuration (la structure) obtiendra la valeur « fort ».
La dernière catégorie, celle du mobilier multi-usage dont I'interprétation reste difficile, autorise l'attribution de la valeur « faible » lorsqu'aucune singularité n'a été relevée et la valeur «moyen » dans le cas contraire.

Pour la notation d'un gisement, nous lui affectons automatiquement la valeur la plus importante attribuée à I'une des structures.

\section{Les gisements}

Parmi les 66 gisements retenus de notre zone d'étude, 31 ont obtenu la valeur « faible », 22 la valeur « moyen » et 13 la valeur « fort » (Fig. 8).

La répartition des gisements par « valeur symbolique » apparaît relativement équilibrée. Une sur-représentation des gisements «faible »au Hallstatt est tout de même à constater. Cela est dû au nombre important de gisements retenus pour cette période n'ayant révélé que des vases de petite taille sans aucune autre configuration remarquable. Ces mêmes données sont localisées sur les cartes de la figure 9. Les trois cartes ci-dessous affichent pour le Hallstatt, la transition entre les deux âges du Fer et La Tène, I'emplacement des gisements retenus en fonction de la valeur du potentiel symbolique qui leur a été attribuée.

Figure 7 :

Diagramme d'attribution d'une « valeur symbolique ». Engel 2013, vol. I, p. 117.

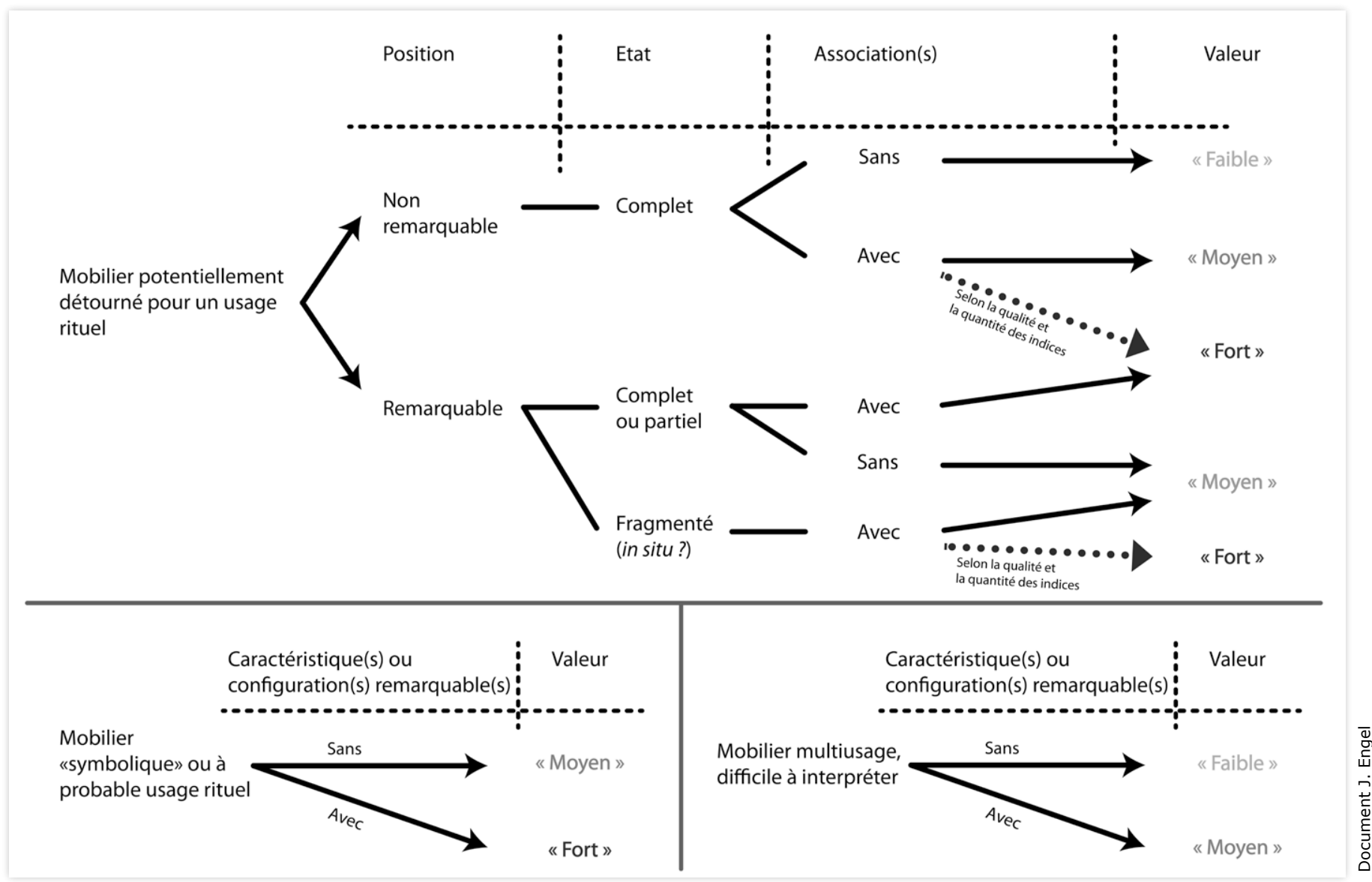


Figure 8 :

Graphique : répartition chronologique des gisements par « valeur symbolique ». Engel 2013, vol. I, p. 119.

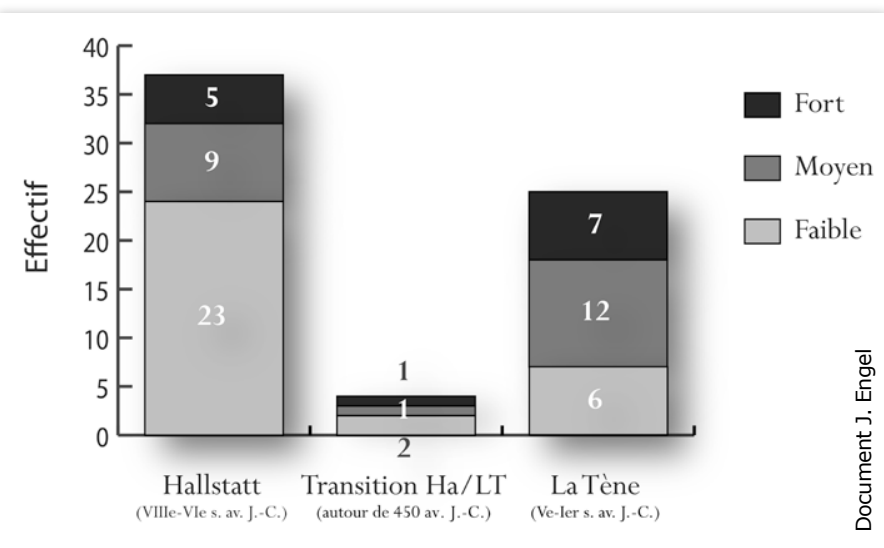

Figure 9 :

Répartition spatiale des gisements par « valeur symbolique ». Engel 2013, vol. I, p. 121. Fond de carte SRTM : http://srtm.csi.cgiar.org.

\section{LES RITUELS}

Les similarités des configurations de notre zone d'étude avec d'autres localisées en Europe celtique, déjà suspectées comme pouvant être le résultat d'un rituel, nous permettent d'identifier des pratiques potentielles. La fiabilité des interprétations proposées devra être évaluée en fonction des valeurs du potentiel symbolique attribuées (Fig. 10).

Nous avons tout d'abord identifié le mobilier de culte, comme les pseudo-kernoi au premier âge du Fer [42] et éventuellement aussi, sans réelle preuve, les vases de petite taille, dans I'hypothèse où ils pouvaient contenir des substances utilisées au cours du rituel. Ensuite, nous avons estimé que certains vestiges pouvaient

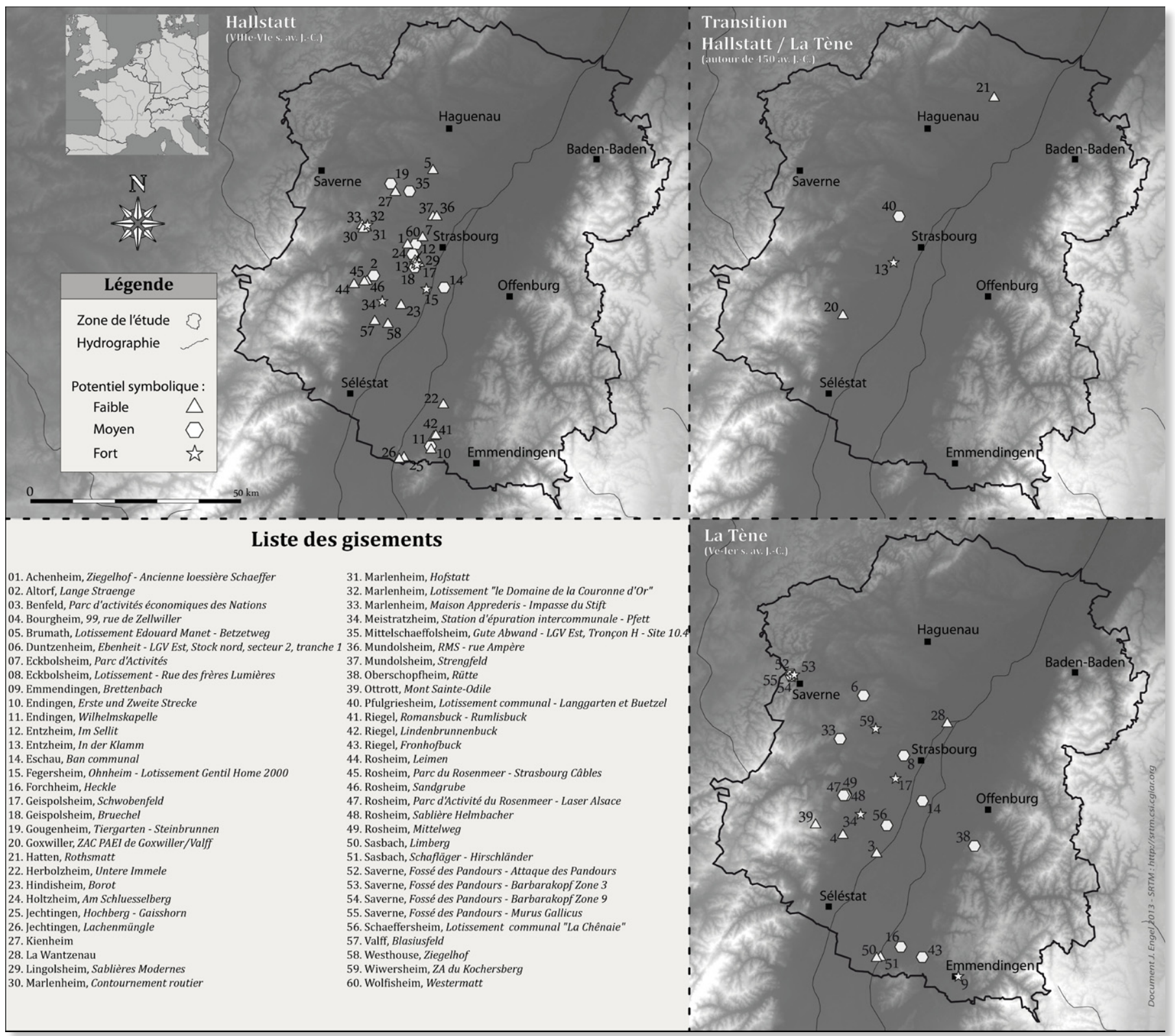


revêtir une valeur symbolique même s'ils ne participaient pas nécessairement à une pratique sacrée. Deux pendeloques datées de La Tène ancienne, une en céramique et l'autre en os (pariétal d'enfant), quatre rouelles de La Tène finale, et des os humains isolés de La Tène finale pourraient correspondre à cette catégorie. Enfin, à partir des configurations les plus remarquables, nous avons identifié de possibles rituels de fondation, de fermeture, mais aussi d'éventuels sacrifices animaux, notamment de chiens et de chevaux, et des trophées. Un ensemble du Hallstatt $D$ témoigne de la pratique du sacrifice, de la consommation et de l'offrande d'un cheval. Au Hallstatt et à La Tène finale, les dépôts de meules associées à des vases dont le contenu non préservé doit être gardé à l'esprit peuvent être des offrandes, peut-être liées aux forces chthoniennes ou encore, au vu de la fonction première du matériel mis en terre, aux cultures ou à la fertilité.

\section{CONCLUSION}

Jusqu'à présent, notre zone d'étude n'a livré que de rares constantes pouvant témoigner de pratiques précises limitées dans le temps et l'espace. Toutefois, le travail d'inventaire préliminaire et l'examen au cas par cas et par marqueur des configurations remarquables auront permis la construction d'une méthode visant à approcher le rituel dans I'habitat, un domaine difficile à appréhender par l'archéologie.

La comparaison de nos résultats avec ceux obtenus dans d'autres régions tend à montrer que les pratiques cultuelles de ce tronçon du fossé rhénan semblent s'intégrer dans celles du monde celtique. Cependant, la question des origines et de la diffusion des pratiques rituelles nécessiterait une étude étendue à une aire géographique plus vaste.

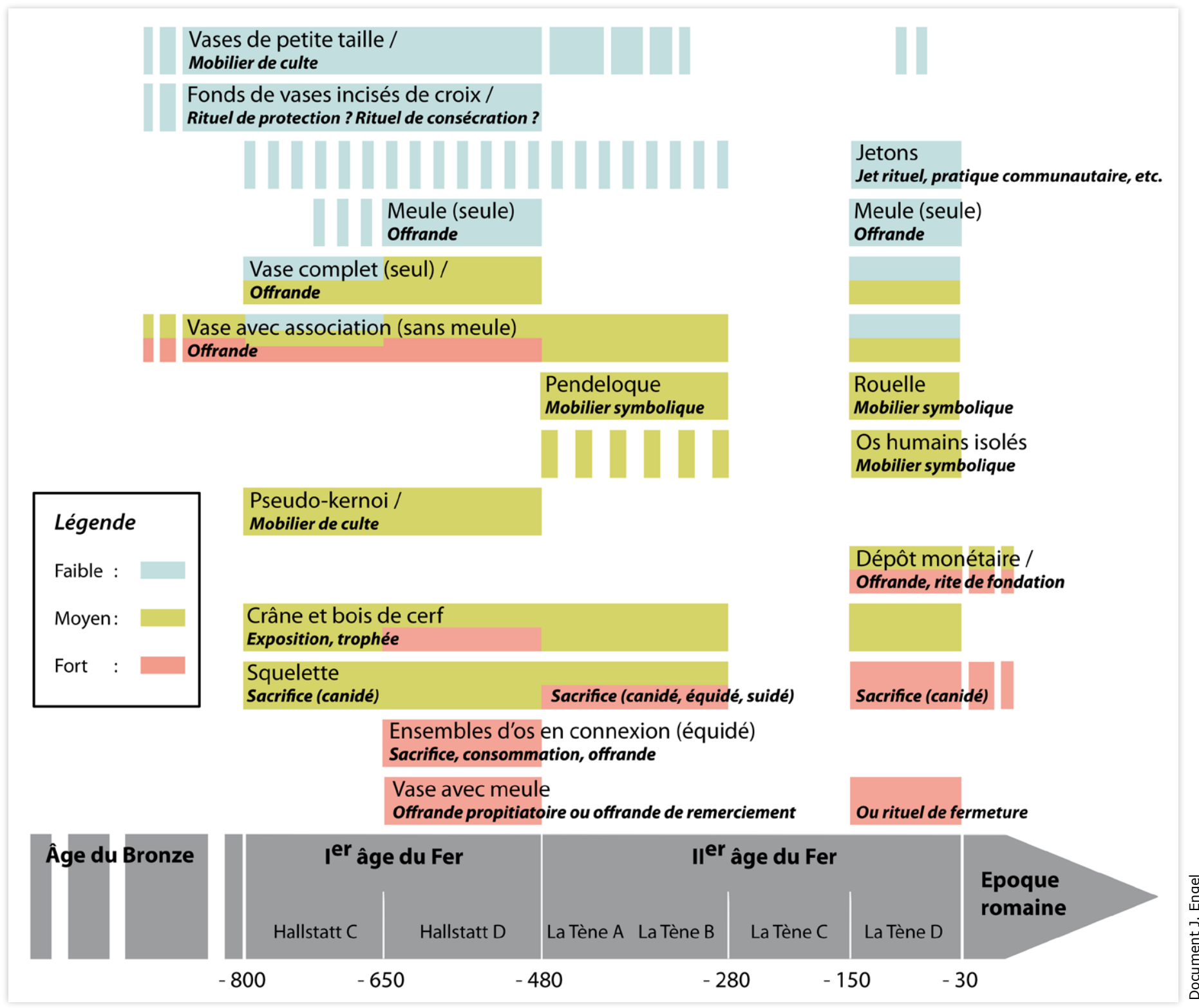


Adam, A.-M., Deffressigne, S., Koenig, M.-P., Lasserre, M., (Éd.), 2011, La céramique d'habitat du Bronze final IIIb à La Tène A en Alsace et en Lorraine : essai de typo-chronologie (Suppl . RAE 29), Dijon, 339 p.

Arcelin, P. \& Brunaux, J.-L., 2003, « Sanctuaires et pratiques cultuelles : I'apport des recherches archéologiques récentes à la compréhension de la sphère religieuse des Gaulois », dans : P. Arcelin \& J.-L. Brunaux(éd.), Cultes et sanctuaires en France à l'âge du Fer, Gallia 60, p. 243-247.

Bernard, L., 2014, «ArkeoGIS v2.0, Éléments d'analyse de la mise en ligne de bases multilingues sur fond cartographique : fonctionnalités, apports et limites », dans : L. Costa, F. Djindjian \& F. Giligny (éd.), Actes des 3es Journées d'Informatique et Archéologie de Paris - JIAP 2012, Paris, 1-2 juin 2012 (Archeologia e Calcolatori, Supplemento 5), Firenze, p. 228-237.

BrunauX, J.-L., 2000, Les religions gauloises. 2e éd. (1 er éd. 1996), Paris, 271 p.

Châtelet, M., Baudoux, J., Dubois S., Fort, B., Gebhardt, A., Henigfeld, Y., Jodry, F., Latron-Colecchia, A., Méniel, P., Mentele, S., Schwartz, D. \& Wiethold, J., 2006, Marlenheim «Maison Apprederis» (Bas-Rhin), Du premier âge du Fer à l'époque médiévale : à l'origine du village actuel, 2 vol., Rapport de fouille [inéd.], INRAP, SRA Alsace.

Dehn, D. \& DrauschKe, J., 2001, « Ein keltischer Goldmünzschatz aus Riegel a. K., Kreis Emmendingen », Archäologische Ausgrabungen in Baden-Württemberg, p. 82-85.

Delattre, V. \& Séguier, J.-M., 2005, « Espaces funéraires et cultuels au confluent Seine-Yonne (Seine-et-Marne) de la fin du ve au $\mathrm{III}^{\mathrm{e}}$ S. av. J.-C. », dans :O. Buchsenschutz, A. Bulard \& TH. Lejars (éd.), XXVI Colloque de I'AFEAF (Suppl. Revue Archéologique du Centre de la France 26), Tours, p. 241-260.

Delattre, V. \& Séguier, J.-M., 2007, « Du cadavre à l'os sec», dans : Ph. Barral, A. Daubigney, C. Dunning, G. Kaenel \& M.-J. Roulière-Lambert (éd.), L'âge du Fer dans l'arc jurassien et ses marges. Dépôts, lieux sacrés et territorialité à l'âge du Fer, Actes du XXIX' colloque international de l'AFEAF ; Bienne 5-8 mai 2005, vol. 2, Besançon, p. 605-620.

DeLnef, H., 2006, « Kernos et pseudo-kernos au Britzgyberg (Illfurth, dép. Haut-Rhin) 》, Archäologisches Korrespondenzblatt, 36, 2, p. 215-224.

Depeyrot, G. \& Piette, J., 2008, Les monnaies et les rouelles du sanctuaire de La Villeneuve-au-Châtelot (Aube). (2 ${ }^{\mathrm{e}}$ siècle avant $\mathrm{J}-\mathrm{C}-5^{\mathrm{e}}$ siècle après J-C) (Moneta 74), Wetteren, $266 \mathrm{p}$.

Engel, J., 2013, Inventaire du potentiel symbolique et rituel dans l'habitat de l'âge du Fer entre Vosges et Forêt-Noire, 2 vol., mémoire de Master sous la direction de L. Bernard, Université de Strasbourg.

FichtL, St., 2003, «Le Fossé des Pandours, un oppidum médiomatrique au Col de Saverne (Bas-Rhin) : état de la question en $1996 »$, dans : S. Plouin \& P. Jud (éd.), Habitats, mobiliers et groupes régionaux à l'âge du Fer, Actes du XXe colloque de l'AFEAF, Colmar-Mittelwihr, 16-19 mai 1996(Suppl. RAE 20), Dijon, p. 189-196.

FichtL, St. \& Adam, A.-M., 1999, L'oppidum médiomatrique du Fossé des Pandours au Col de Saverne (Bas-Rhin) : rapport triennal 1997-1999, Rapport de fouille programmée, UMB, SRA Alsace, $178 \mathrm{p}$.

FLeisCher, F. \& LANDOLT, M., 2012, « Entzheim-Geispolsheim (Bas-Rhin, Frankreich) - Zur Entwicklung einer Siedlungslandschaft in der frühen Eisenzeit », dans : C. Tappert, Chr. Later, J. Fries-Knoblach, P. C. Ramsl, P. Trebsche, S. Wefers \& J. Wiethold (éd.), Wege und Transport, Beiträge zur Sitzung der AG Eisenzeit während der 80. Verbandstagung des West- und Süddeutschen Verbandes für Altertumsforschung e.V. in Nürnberg 2010 (Beiträge zur Ur- und Frühgeschichte Mitteleuropas 69), Halle/Sa, p. 193-207.

Fleischer, F., LANdolt, M. \& Roth-Zehner, M., 2013, « Die eisenzeitlichen Siedlungsbestattungen des Elsass », dans : N. MüllerScheeßel (éd.), Irreguläre Bestattungen in der Urgeschichte: Norm, Ritual, Strafe ... ?, Akten der Internationalen Tagung in Frankfurt a. M. vom 3. bis 5. Februar 2012, Bonn, p. 439-470.

Gransar, F., Auxiette, G., Desenne, S., Henon, B., François, M., Matterne, V. \& Pinard, E., 2007, « Expressions symboliques, manifestations rituelles et cultuelles en contexte domestique au $\mathrm{I}^{\mathrm{ER}}$ millénaire avant notre ère dans le Nord de la France », dans : Ph. Barral, A. Daubigney, C. Dunning, G. Kaenel \& M.-J. Roulière-Lambert(éd.), L'âge du Fer dans l'arc jurassien et ses marges. Dépôts, lieux sacrés et territorialité à l'âge du Fer, Actes du XXIX'e colloque international de l'AFEAF ; Bienne 5-8 mai 2005, vol. 2, Besançon, p. 549-564.

Gruel, K., 2007, « De la nature des dépôts monétaires gaulois », dans : Ph. Barral, A. Daubigney, C. Dunning, G. Kaenel \& M.-J. Roulière-Lambert (éd.), L'âge du Fer dans l'arc jurassien et ses marges. Dépôts, lieux sacrés et territorialité à l'âge du Fer, Actes du XXIXe colloque international de l'AFEAF ; Bienne 5-8 mai 2005, vol. 2, Besançon, p. 709-714.

Jodry, Fl. \& FéLIU, CL., 2009, « Nouvelles données sur les dépôts de meules rotatives : deux exemples de La Tène finale en Alsace », dans : S. Bonnardin, C. Hamon, M. Lauwers \& B. Quilliec (éd.), Du matériel au spirituel : réalités archéologiques et historiques des «dépôts» de la Préhistoire à nos jours, Actes des Rencontres, 16-18 octobre 2008, Antibes, p. $275-282$.

Kuhnle, G., Schneikert, F. \& Zehner, M., 1998, « Deux puits profonds de La Tène finale sur les terrasses de lœss à l'ouest de Strasbourg : Holtzheim «Les Abattoirs» 1994 et Eckbolsheim «Lotissement» 1996 », Cahiers Alsaciens d'Archéologie d'Art et d'Histoire 61, p. 43-51.

Landolt, M., Millet, E., Roth-Zehner, M., avec la collab. de Barrand, H., Cartier, E., Mauduit, A. \& Putelat, O., 2010, «Pratiques funéraires en Alsace du ve au Ier siècle avant J.-C », dans :Ph. Barral, B. Dedet, F. Delrieu, P. Giraud, I. Le Goff, S. Marion \& A. Villard-Le Tiec (éd.), Gestes funéraires en Gaule au Second âge du Fer, Actes du XXXIII colloque international de I'AFEAF, Caen, 20-24 mai 2009, Besançon, p. 207-230. 
Landolt, M., avec la contrib. de Adam, A.-M., Alix, G., Charrié, A., Ferrier A., Fleischer, F., Francisco, S., Gratuze, B., Leprovost, C., Millet, E., Paleau, L., Pélissier, A., Putelat, O., Roth-Zehner, M. \& SchaAl, C., 2013, Entzheim-Geispolsheim, Bas-Rhin, Lotissement d'activités du quadrant 4, Entzheim «In der Klamm» et Geispolsheim « Schwobenfeld», vol. 3 : Les occupations protohistoriques, Rapport de fouille préventive, PAIR, SRA Alsace, $602 \mathrm{p}$.

LANDOLt, M., avec la collab. de Fleischer, F., à paraître, « Nouveaux éléments sur la chronologie, la répartition et l'interprétation des kernoi et pseudo-kernoi en Europe tempérée à l'Âge du fer», dans : D. Vitali (éd.), Les Celtes dans le Nord de l'Italie, Actes du XXXVIe colloque de l'AFEAF de Vérone, 2012, Dijon.

LANGE, G., 1983, Die menschlichen Skelettreste aus dem Oppidum von Manching (Die Ausgrabungen in Manching, Band 7), Wiesbaden, $190 \mathrm{p}$.

Normand, B., 1973, L'Âge du Fer en Basse-Alsace (Publication de la Société Savante d'Alsace et des régions de I'Est, XIV).

Peyre, Chr., 2000, «Documents sur l'organisation publique de l'espace dans la cité gauloise : le site de Villeneuve-Saint-Germain et la bilingue de Verceil », dans : St.Verger (éd.), Rites et espaces en pays celte et méditerranéen : études comparées à partir du sanctuaire d'Acy-Romance, Table-ronde organisée à Rome les 18 et 19 avril 1997 (Collection de l'École française de Rome 276), Rome, p. $155-206$.

Ropiot, V. \& MAZière, F., 2007, «Témoins d'activités cultuelles en Languedoc occidental, en Roussillon et en Ampourdan protohistoriques (IX ${ }^{\mathrm{e}}-\mathrm{III}^{\mathrm{e}}$ S. av. n. è.) », », dans : Ph. Barral, A. Daubigney, C. Dunning, G. Kaenel \& M.-J. Roulière-Lambert (éd.), L'âge du Fer dans l'arc jurassien et ses marges. Dépôts, lieux sacrés et territorialité à l'âge du Fer, Actes du XXIX colloque international de I'AFEAF ; Bienne 5-8 mai 2005, vol. 2, Besançon, p. 731-749.

RottländeR, R.C. A., 1985, « Chemische Untersuchungen an Keramischen Funden von der Heuneburg, Gemeinde Herbertingen, Kreis Sigmaringen », Fundberichte aus Baden-Württemberg 10, p. 19-27.

SAlicis, C., 1996, Rouelles et anneaux et objets singuliers (Institut de préhistoire et d'archéologie des Alpes-Maritimes - IPAAM), Nice, $167 \mathrm{p}$.

VAN Den Boom, H., 1989, Keramische Sondergruppen der Heuneburg (Heuneburgstudien 7, Römisch-germanische Forschungen 47), Berlin, p. 1-134. 